\title{
Feasibility of detecting myocardial ischemia using first-pass contrast MRI and regadenoson
}

\author{
Matthew Lyons ${ }^{1}$, Cylen Javidan-Nejad ${ }^{2}$, Ibrahim M Saeed ${ }^{3}$, Donna Lesniak ${ }^{2}$, Gary R McNeal ${ }^{4}$, Agus Priatna ${ }^{4}$, \\ Robert J Gropler ${ }^{2}$, Pamela K Woodard ${ }^{2^{*}}$ \\ From 15th Annual SCMR Scientific Sessions \\ Orlando, FL, USA. 2-5 February 2012
}

\section{Summary}

A single injection of regadenoson can be used instead of an adenosine infusion to produce coronary vasodilatation and demonstrate myocardial ischemia during firstpass perfusion cardiac MRI.

\section{Background}

Cardiac stress MR perfusion imaging requires an MRI compatible infusion pump for the administration of adenosine or a non-MRI compatible pump housed in the control room or beyond the 10-Gauss line. Regadenoson is a recently FDA-approved A2A receptor agonist that can be given intravenously in a single bolus. It has been shown to provide diagnostic information regarding myocardial ischemia on SPECT-MPI.

\section{Methods}

42 patients (34 M, 55 yrs, range $41-73$ yrs) with a reversible myocardial perfusion defect on SPECT-MPI underwent a cardiac perfusion MRI within 7 days of the SPECT-MPI. MR exams consisted of short and long axis cine steady state free precession (SSFP) imaging, matched gradient-recalled echo (GRE) GRAPPA temporal parallel acquisition (TPAT) first-pass stress perfusion (TR $2.3 \mathrm{msec}$, TE $1.1 \mathrm{msec}, 80 * 256$ matrix, $1.4 \mathrm{x}$ $3.1 \mathrm{~mm} 2$ ), and delayed contrast-enhanced (DCE) T1 GRE imaging. First-pass perfusion images were obtained 30 seconds after regadenoson 400 micrograms administered in a single IV bolus and during power injection of $0.075 \mathrm{mmol} / \mathrm{Kg}$ of gadobenate dimeglumine at $5 \mathrm{~mL} / \mathrm{sec}$ IV followed by normal saline flush. DCE imaging was obtained 10 minutes after injection of an additional $0.025 \mathrm{mmol} / \mathrm{Kg}$ of contrast agent.

${ }^{2}$ Washington University School of Medicine, St. Louis, MO, USA

Full list of author information is available at the end of the article

\section{Results}

All but one patient tolerated the regadenoson MR examination. One patient had chest pain shortly after imaging, and received aminophylline, with resolution of symptoms. MR showed ischemia in 33/42 subjects. In 8 subjects the MR perfusion exam was normal. Five of these 8 patients underwent clinically-ordered invasive cardiac catheterization (ICA) within 3-18 days of the MRI examination. ICA showed no stenoses, suggesting SPECT attenuation artifact. The other $3 / 8$ patients had no MACE within 30-180 days. In one patient, SPECT demonstrated ischemia only, while MRI showed infarct only in the same segment.

\section{Conclusions}

Regadenoson can be used in cardiac MR perfusion imaging to demonstrate ischemia. MRI perfusion imaging may be useful in differentiating attenuation artifact from true disease.

\section{Funding}

Funding for this project was provided by Astellas. Additional research support provided by Siemens Medical Systems.

\section{Author details \\ ${ }^{1}$ Metrohealth Medical Center, Cleveland, OH, USA. ${ }^{2}$ Washington University School of Medicine, St. Louis, MO, USA. ${ }^{3}$ St. Luke's Cardiovascular Consultants, Kansas City, MO, USA. ${ }^{4}$ Siemens Medical Systems, Malvern, PA, USA}

Published: 1 February 2012

doi:10.1186/1532-429X-14-S1-P11

Cite this article as: Lyons et al.: Feasibility of detecting myocardial ischemia using first-pass contrast MRI and regadenoson. Journal of Cardiovascular Magnetic Resonance 2012 14(Suppl 1):P11. 